\title{
Investigations on Vehicle Rollover Prevention Using LQG Regulator
}

\author{
Binda Mridula Balakrishnan ${ }^{1}$ and Marimuthu Rajaram ${ }^{2}$ \\ ${ }^{1}$ Sathyabama University, Chennai 119, India \\ ${ }^{2}$ Anna University, Chennai 25, India \\ Correspondence should be addressed to Marimuthu Rajaram; rajaramgct@rediffmail.com
}

Received 18 August 2014; Revised 5 November 2014; Accepted 6 November 2014; Published 9 December 2014

Academic Editor: Alkesh Punjabi

Copyright (c) 2014 B. Mridula Balakrishnan and M. Rajaram. This is an open access article distributed under the Creative Commons Attribution License, which permits unrestricted use, distribution, and reproduction in any medium, provided the original work is properly cited.

\begin{abstract}
This paper presents results of an initial investigation into vehicle roll model and control strategies suitable for preventing vehicle untripped rollovers. For vehicles that are deemed to be susceptible to wheel-liftoff, various control strategies are implemented in simulation. In this study, the authors propose a method for rollover prevention that does not require such accurate contact information. The validity of the stability margin is shown, and it is used to realize rollover prevention in the direction of the roll. The primary assumption in their implementation is that the vehicle in question is equipped with a conventional controller system.
\end{abstract}

\section{Introduction}

The main innovations of this paper are (1) safety concerns, (2) understanding the physics behind rollover, and (3) benefit to the academic community.

Vehicle rollover is a public safety issue [1-3]. According to the National Highway Traffic Safety Administration's (NHTSA) National Center for Statistics and Analysis, from 1991 to 2001 the number of passenger vehicle occupants killed in all motor vehicle crashes increased $4 \%$, while fatalities in rollover crashes increased 10\%. In 2001 alone, 10,138 people died in rollover crashes, a figure that represents $32 \%$ of occupant fatalities for the year $[4,5]$.

To help address this public safety issue, impending vehicle rollover needs to be detected and mitigated by activating chassis control actuators based on the vehicle rollover conditions [6, 7]. Vehicle rollover-avoidance systems perform best when the rollover conditions are determined in a timely manner. Timely determination of a pending vehicle rollover requires measurement of roll rate and roll angle. For example, a rollover-avoidance system using differential braking by detecting wheel-liftoff or lateral acceleration exceeding a predetermined threshold is presented in $[8,9]$. A time-torollover (TTR) metric for rollover prevention is shown in
$[1,10]$. TTR provides an assessment of impending rollover. A method of rollover detection based on the roll rate and roll angle phase plane is illustrated in [11]. The combined use of a roll rate sensor and roll angle estimate with threeaxis accelerometers to classify rollover events is discussed in [12]. All of these methods use some form of thresholds for roll rate and roll angle magnitudes to detect an impending vehicle rollover. However, the majority of production vehicles are not equipped with roll angle or roll rate sensors. Since friction-induced vehicle rollover is related to both the yaw and roll dynamics, a rollover-avoidance algorithm needs an estimator for both roll angle and roll rate in addition to the production yaw sensor. Furthermore, the prediction of an impending rollover must include the effects of roll stiffness due to the travel limit on the wheel suspension. These effects are neglected in [13]. A model for predicting vehicle resistance to rollover is discussed in [14].

The automobile is a highly nonlinear dynamic system containing many parameters that are either difficult or (as yet) impossible to directly measure. While many physical parameters such as mass, location of mass center, and inertial properties have been made available by the National Highway Traffic Safety Administration, many common parameters used in vehicle roll models such as suspension stiffness 
and damping characteristics are not readily available to the experimenter. This makes it difficult or impossible to perform a broad vehicle rollover study that encompasses multiple vehicles without actually having access to the vehicles themselves. A parameter of significant importance to the study of vehicle rollover that is often overlooked is the driver and his/her reactions to the world. While a number of mathematical representations of the human driver have been developed [9-13], none of them can remove the uncertainty of life and predict exactly how a driver will respond in an emergency situation. Therefore, any study of rollover must allow for all possible steering responses.

There are two options to the automobile designer wishing to design a vehicle incapable of rollover: to design a vehicle chassis or chassis control system very conservatively to mitigate driver inputs that push the vehicle to the limits of safe handling and instability or modify the driver's input to the vehicle such that instability is avoided.

In the past, auto manufacturers have sought chassis modifications as a means to influence stability. This is evidenced by the appearance of various types of active suspensions, electronic stability controls, and traction control systems in modern production vehicles. But recent research is changing focus toward developing new types of assistive devices.

For example, research is currently underway to predict, for measured steering inputs, the amount of time that will elapse before wheel-lift will occur. Such predictive methods are developed in order to either provide a warning to drivers or utilize on-board systems to prevent rollover onset. Examples of the development of time-to-rollover (TTR) metrics may be found by Chen, working under Huei Peng at the University of Michigan Transportation Research Institute (UMTRI) [15-18], and Hyun, working under Reza Langari at Texas A\&M.

The most apparent drawback to such methods is the limited amount of warning given. It was found by Chen that even under ideal circumstances, the largest forward prediction in time and hence the largest amount of warning a driver can feasibly use is 0.3 seconds when considering an SUV [8]. Such warnings are barely at the limit of human reaction times for unexpected events. Thus, there is a clear need for and utility in an automated system capable of preventing vehicle roll by modifying a vehicle's steering input. Additionally, current TTR methods primarily rely on setting a threshold for roll angle and/or lateral acceleration. Despite extensive model development by academic researchers, no one has yet been able to consistently match in simulation the rollover threshold predictions found by the NHTSA experimental trials. Whether a model is used to predict the amount of time before this limit is reached or sensors are used to determine when this limit has been crossed and a TTR is then generated, the lack of simulation-based tools reflects the current lack of understanding of the physics behind vehicle rollover. The option of allowing a vehicle to actively modify the driver's input is an idea only in its infancy, but there are research precedents. One example is presented by Hosaka and Morakami from Keio University, Japan, in [17]. Here, a combination of PD control, state feedback control, and a disturbance observer was used to either provide additional steering input to or directly modify a driver's steering command to deal with disturbances such as wind gusts for increased yaw stability. Another example is by $\mathrm{Oh}$ et al., from Hyundai and associated with Hanyang University [18].

Here the authors propose a feedforward control method that imposes an artificial gear ratio on the steering input so that the dynamic behavior of the vehicle may be changed according to the situation. It also discusses the possibility to prevent rollover by not allowing a driver to steer the vehicle into a region where the lateral acceleration is above a certain threshold. Additionally, the system would add more resistive torque to the wheel as the driver approaches this limit, making it more difficult to steer. Other examples may be found related to increasing the stability of four-wheel steered vehicles as investigated by Ackermann while being at the Institute for Robotics and System Dynamics [19] and Plochl at the Institute of Mechanics at the University of Technology in Wien, Austria [4]. Additionally, work done by J. Christian Gerdes at the Dynamic Design Laboratory at Stanford University includes methods that allow improved disturbance rejection to vehicle steering angle [20] and roll limiting techniques which use a combination of a steer-bywire system and measurements from a Global Positioning System (GPS) and an inertial measurement unit (IMU) to ensure tracking of the desired path. Despite these findings, the literature remains rather limited with regard to this area.

This paper seeks to contribute significantly to the rollover mitigation methods that directly modify the driver's steering command in a way such that the driver does not realize the effect of the controller. However, in order to properly accomplish this goal, the physics behind vehicle rollover must first be understood.

This paper deals with standardized SAE vehicle axis system. The section also explains the three-degree-of-freedom vehicle model. PID controller system is presented and also explains the need of this controller. The results concerning the aim in this paper are to suggest a solution to the problem of an initial investigation into models and control strategies suitable for predicting and preventing vehicle rollover due to untripped driving maneuvers.

\section{Rollover Model}

All numerical representations follow the standard SAE right-handed sign convention utilizing the simplified threefreedom vehicle model as depicted in Figure 1.

In Figure 1(a), $x-y$ plane, the lateral forces over the front and rear tires are denoted by $F_{y 1}$ and $F_{y 2}$. The sideslip angle $\beta$ and the roll angle $\phi$ are assumed to be minor. The total forces and torques over the whole vehicle are given by

$$
\begin{gathered}
\sum F_{y}=F_{y 1} \cos \delta+F_{y 2}=m a_{y}, \\
\sum M_{z}=a F_{y 1} \cos \delta-b F_{y 2}=I_{z} \dot{\omega}_{r},
\end{gathered}
$$

where $m$ is the total mass of the vehicle, the lengths $a$ and $b$ are defined as shown in Figure 1(a), $a_{y}$ is the lateral acceleration, 


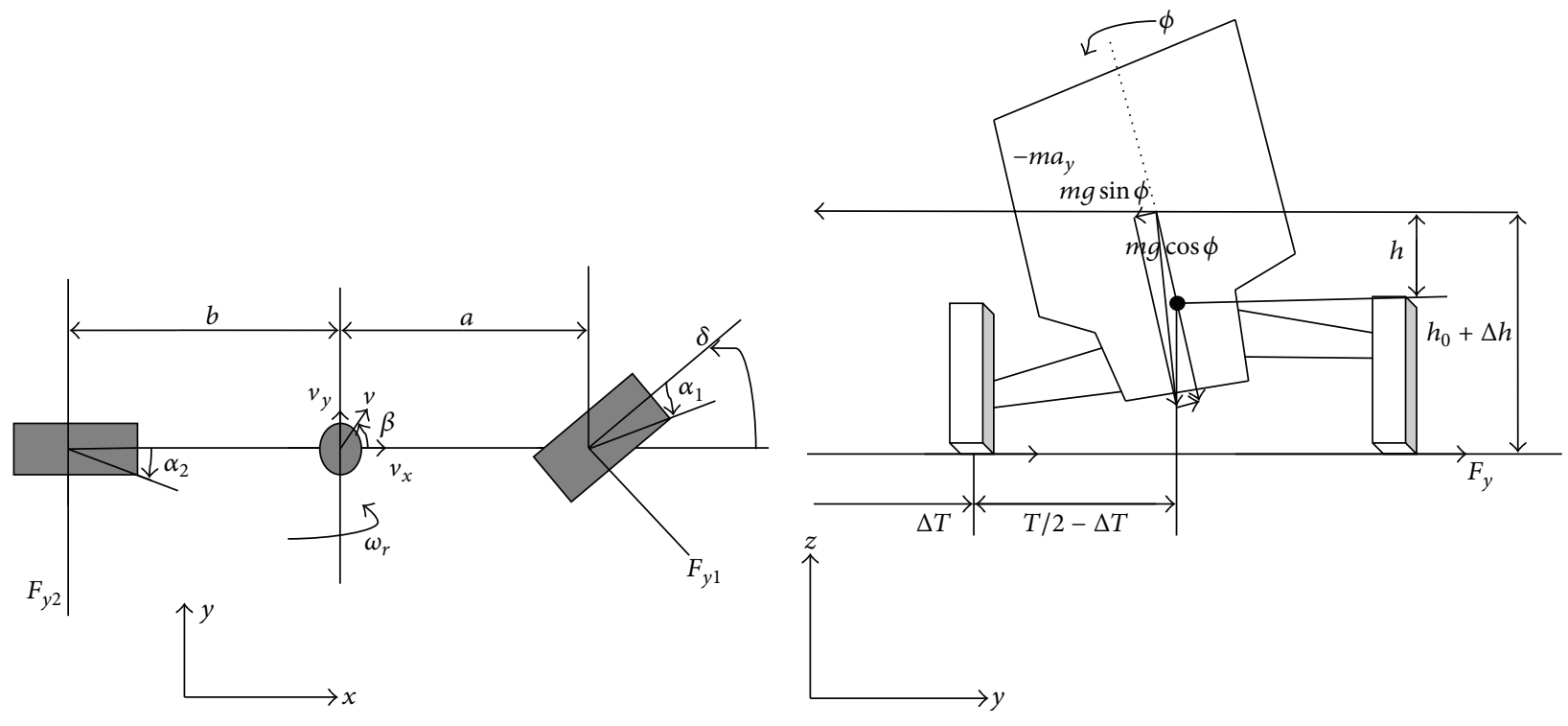

(a) $x-y$ of the simplified model

(b) $y-z$ of the simplified model

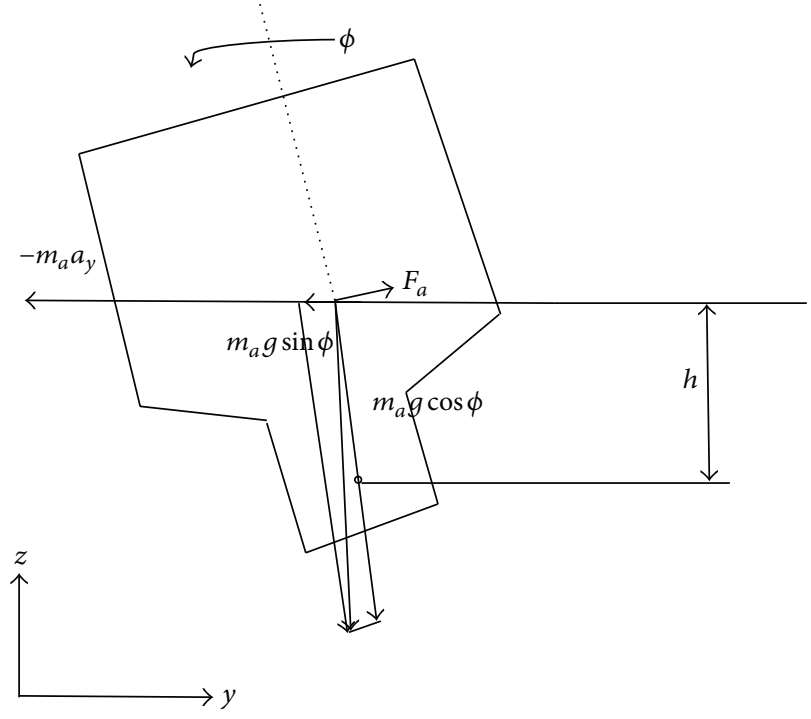

(c) The vehicle body model

Figure 1: Simplified vehicle rollover model.

$\dot{\omega}_{r}$ is the yaw acceleration, and $I_{z}$ is the yaw moment of the vehicle inertia.

In addition, the lateral forces $F_{y 1}=k_{1} \alpha_{1}$ and $F_{y 2}=k_{2} \alpha_{2}$, where $k_{1}$ and $k_{2}$ are the cornering stiffness of front and rear tires, respectively, $\alpha_{1}$ and $\alpha_{2}$ are the slip angles of front and rear tires, $\alpha_{1}=\left(\beta+a \omega_{r} / v_{x}\right)-\delta, \alpha_{2}=\left(\beta-b \omega_{r} / v_{x}\right), v_{x}$ is the longitudinal velocity, and $\omega_{r}$ is the yaw rate. Consequently, the following equation is derived:

$$
\begin{gathered}
a_{y}=\frac{\left(k_{1}+k_{2}\right) \beta}{m}+\frac{\left(a k_{1}-b k_{2}\right) \omega_{r}}{m v_{x}}-\frac{k_{1} \delta}{m}, \\
\dot{\omega}_{r}=\frac{\left(a k_{1}-b k_{2}\right) \beta}{I_{z}}+\frac{\left(a^{2} k_{1}+b^{2} k_{2}\right) \omega_{r}}{I_{z} v_{x}}-\frac{a k_{1} \delta}{I_{z}} .
\end{gathered}
$$

In Figure 1(b), $y$ - $z$ plane, the total forces and torques over the whole vehicle are given by

$$
\begin{gathered}
\sum F_{z}=F_{n 1}+F_{n 2}-m g=0, \\
\sum M_{x}=m g h \sin \phi+m a_{y}\left(h_{0}+\Delta h-h\right) \\
+F_{n 2}\left(\frac{T}{2}+\Delta T\right)-F_{n 1}\left(\frac{T}{2}-\Delta T\right) \\
=I_{x} \ddot{\phi},
\end{gathered}
$$

where $\ddot{\phi}$ is the body roll acceleration, $h_{0}$ is the height of the vehicle's center of gravity (CG) standing above the ground level, $h$ is the distance between the vehicle CG and the assumed roll axis, $T$ is the width of the vehicle track, and $\Delta h$ 
and $\Delta T$ are the deformations of suspensions and tires. $F_{n 1}$ and $F_{n 2}$ are normal forces over the left and right wheels, and $I_{x}$ is the roll moment of vehicle inertia.

The restoring force $F_{a}$ in Figure 1(c) is associated with the roll movement, $F_{a}=\left(k_{\Phi} \phi+c_{\Phi} \dot{\phi}\right) / h$, where $k_{\Phi}$ and $c_{\Phi}$ are the total roll stiffness and roll damping of suspensions, and $\dot{\phi}$ is the roll rate.

The total torques over the body with respect to the roll axis are given by

$$
\sum M_{x s}=m_{s} a_{y} h+m_{s} g h \sin \phi-F_{a} h=I_{x s} \ddot{\phi},
$$

where $m_{s}$ is the sprung mass that represents the total vehicle mass $m$ excluding the suspension and tire and $I_{x s}$ is the roll moment for the inertia of the sprung mass.

Compared with the sprung mass, the unsprung mass is much smaller. Therefore, we can suppose that $m_{s}=m$ and $I_{x s}=I_{x}$ and establish (5) as follows:

$$
\begin{gathered}
\ddot{\phi}=\frac{m h\left(a_{y}+g \sin \phi\right)}{I_{x}}-\frac{k_{\phi} \phi}{I_{x}}-\frac{c_{\phi} \dot{\phi}}{I_{x}}, \\
F_{n 1}-F_{n 2}=\frac{2 m a_{y}\left(h_{0}+\Delta h-h\right)}{T}+\frac{2 m g \Delta T}{T} \\
-\frac{2\left(k_{\phi} \phi+c_{\phi} \dot{\phi}\right)}{T}, \\
F_{n 1}+F_{n 2}=m g, \\
\operatorname{LTR}=\left(\frac{F_{n 1}-F_{n 2}}{F_{n 1}+F_{n 2}}\right),
\end{gathered}
$$

where LTR stands for the load transfer ratio, an important criterion to evaluate the vehicle status.

Since $a_{y}=\dot{v}_{y}+v_{x} \omega_{r}, \beta=v_{y} / v_{x}$, after introducing the state vector $x=\left[\begin{array}{llll}v_{y} & \omega_{r} & \dot{\phi} & \phi\end{array}\right]^{T}$, the motions of this model can be described by

$$
\dot{x}=A x+B \delta,
$$

where

$$
\begin{gathered}
A=\left[\begin{array}{cccc}
\frac{k_{1}+k_{2}}{m v_{x}} & \frac{a k_{1}-b k_{2}}{m v_{x}}-v_{x} & 0 & 0 \\
\frac{a k_{1}-b k_{2}}{I_{z} v_{x}} & \frac{a^{2} k_{1}+b^{2} k_{2}}{I_{z} v_{x}} & 0 & 0 \\
\frac{k_{1}+k_{2}}{I_{x} v_{x}} & \frac{a k_{1}-b k_{2}}{I_{x} v_{x}} & \frac{-c_{\phi}}{I_{x}} & \frac{m g h-k_{\phi}}{I_{x}} \\
0 & 0 & 1 & 0
\end{array}\right], \\
B=\left[\begin{array}{cccc}
\frac{-k_{1}}{m} & \frac{-a k_{1}}{I_{z}} & \frac{-k_{1}}{I_{z}} & 0
\end{array}\right]^{T} .
\end{gathered}
$$

\section{Rollover Prevention Method by Mitigation Control}

The running vehicle intends to overshoot in its response to a suddenly applied lateral acceleration. Therefore, the inner wheels may have lifted off during the steering when the applied acceleration is lower than the nominal permit value that is in compliance with the steady state laws.

From (5), we can see

$$
\ddot{\phi}=\frac{m h\left(a_{y}+g \sin \phi\right)}{I_{x}}-\frac{k_{\phi} \phi}{I_{x}}-\frac{c_{\phi} \dot{\phi}}{I_{x}}
$$

where the roll angle $\phi$ is small enough to get $\sin \phi \approx \phi$, and after applying Laplace law to the aforementioned differential equation, we established the following new equation:

$$
\frac{\phi(s)}{a_{y}(s)}=\frac{\left(k_{\phi}-m g h\right) / I_{x}}{s^{2}+\left(c_{\phi} / I_{x}\right) s+\left(k_{\phi}-m g h\right) / I_{x}} \cdot \frac{m h / I_{x}}{\left(k_{\phi}-m g h\right) / I_{x}},
$$

where the angular frequency is

$$
\omega_{n}=\left[\frac{\left(k_{\phi}-m g h\right)}{I_{x}}\right]^{1 / 2}
$$

and the damping ratio is

$$
\xi=\frac{c_{\phi}}{2\left[\left(k_{\phi}-m g h\right) I_{x}\right]^{1 / 2}} .
$$

When the input $a_{y}$ is set to be the unit step function, the overshoot is

$$
M_{P}=\exp \left(-\frac{\xi \pi}{\sqrt{\left(1-\xi^{2}\right)}}\right)
$$

At this point, we found that vehicle dynamics is determined by the first part of (9), whereas the second part defines the vehicle steady state. These findings form the base of the mitigation control referred to in the rest of this section.

A specific brand of midsize SUV was used as the test vehicle in this study, of which the relevant parameters are shown in Table 1.

With the rollover model and vehicle parameters, we achieved the damping ratio of the original vehicle, $\xi=0.71$, and the overshoot value of the roll angle at $38.18 \%$ as shown in Figure 9. Thus, the damping ratio needs to be raised in order to reduce the overshoot value. In other words, we can improve the restoring force $F_{a}$ by increasing the roll stiffness $k_{\Phi}$ or the roll damping $c_{\Phi}$. However, it needs to bear in mind that the roll stiffness $k_{\Phi}$ and the roll damping $c_{\Phi}$ must be increased simultaneously. Otherwise, the damping ratio $\xi$ may be decreased.

With the rollover model and vehicle parameters, we improve the damping ratio of the vehicle, $\xi=0.95$, and the overshoot value of the roll angle at $0.71 \%$ as shown in Figure 10. 
TABLE 1

\begin{tabular}{lc}
\hline Parameters & Values \\
\hline$m$ & $1030 \mathrm{Kg}$ \\
$I_{y y}$ & $1705 \mathrm{Kg}-\mathrm{m}^{2}$ \\
$m_{s}$ & $825 \mathrm{Kg}$ \\
$I_{x x}$ & $375 \mathrm{Kg}-\mathrm{m}^{2}$ \\
$I_{x z}$ & $72 \mathrm{Kg}-\mathrm{m}^{2}$ \\
$I_{z z}$ & $1850 \mathrm{Kg}-\mathrm{m}^{2}$ \\
$a$ & $0.93 \mathrm{~m}$ \\
$b$ & $1.56 \mathrm{~m}$ \\
$L$ & $1.4 \mathrm{~m}$ \\
$h$ & $0.52 \mathrm{~m}$ \\
$k_{1}$ & $-45500 \mathrm{~N} / \mathrm{rad}$ \\
$k_{2}$ & $-76650 \mathrm{~N} / \mathrm{rad}$ \\
$k_{\varphi}$ & $53000 \mathrm{~N}-\mathrm{m} / \mathrm{rad}$ \\
$C_{\varphi}$ & $6000 \mathrm{~N}-\mathrm{m}-\mathrm{sec} / \mathrm{rad}^{2}$ \\
\hline
\end{tabular}

\section{PID Controller, GA Tuned PID Controller, and LQG Regulator}

4.1. PID Controller. The outputs for the three terms, proportional, integral, and derivative terms, are summed to calculate the output of the PID controller. First estimation is the equivalent of the proportional action of a PID controller. The integral action of a PID controller can be thought of as gradually adjusting the output when it is almost right. Derivative action can be thought of as making smaller and smaller changes as one gets close to the right level and stopping when it is just right, rather than going too far. Making a change that is too large when the error is small is equivalent to a high gain controller and will lead to overshoot.

If the controller were to repeatedly make changes that were too large and repeatedly overshoot the target, this control loop would be termed unstable and the output would oscillate around the set point in either constant, a growing, or a decaying sinusoid. A human would not do this because they are adaptive controllers, but PID controllers do not have the ability to learn and must be set up correctly. Selecting the correct gains for effective control is known as tuning the controller.

A PID is the most commonly used feedback controller. A PID controller calculates error value as the difference between a measured process variable and a desired set point. The controller attempts to minimize the error by adjusting the process control inputs. The PID controller calculation (algorithm) involves three-term control: proportional, integral, and derivative values denoted by $\mathrm{P}, \mathrm{I}$, and $\mathrm{D}$. These values can be interpreted in terms of time.

(i) P depends on the present error.

(ii) I depends on the accumulation of past errors.

(iii) $\mathrm{D}$ is prediction of future errors.

Based on current rate of change, some applications may require using only one or two actions to provide the appropriate system control. This is achieved by setting the other parameters to provide the appropriate system control. This is achieved by settling the other parameters to zero.

(i) The proportional term: providing an overall control action proportional to the error signal through the all pass gain factor.

(ii) The integral term: reducing the steady state errors through low-frequency compensation by an integrator.

(iii) The derivative term: improving transient response through high frequency compensation by a differentiator.

A PID controller will be called a PI, PD, P, or I controller in the absence of the respective control actions. PI controllers are fairly common and eliminate the steady state error. After certain limit increase overshoot reduces rise time. Since derivative action is sensitive to measurement noise, the absence of an integral term may prevent the system from reaching its target value due to the control action. The PID control scheme is named after its three correcting terms, whose sum constitutes the manipulated variable (MV). The proportional, integral, and derivative terms are summed to calculate the output of the PID controller.

PID controllers have traditionally been chosen by control system engineers due to their flexibility and reliability. A PID controller has proportional, integral, and derivative terms that can be represented in transfer function form as

$$
K(s)=K_{p}+\frac{K_{i}}{s}+K_{d} s
$$

where $K_{p}$ is proportional gain, a tuning parameter, $K_{i}$ is integral gain, a tuning parameter, and $K_{d}$ is derivative gain, a tuning parameter.

By tuning these PID controller gains, the controller can provide control action designed for specific process requirements. The proportional term drives a change to the output that is proportional to the current error. This proportional term is concerned with the current state of the process variable. The integral term $\left(K_{i}\right)$ is proportional to both the magnitude of the error and the duration of the error. It (when added to the proportional term) accelerates the movement of the process towards the set point and often eliminates the residual steady state error that may occur with a proportional only controller.

4.1.1. Optimizing of PID Controller. For the system under study, Ziegler-Nichols tuning rule based on critical gain $K_{e r}$ and critical period $P_{e r}$ will be used. In this method, the integral time $T_{i}$ will be set to infinity and the derivative time $T_{d}$ to zero. This is used to get the initial PID setting of the system.

In this method, only the proportional control action will be used. The $K_{p}$ will be increased to a critical value $K_{e r}$ at which the system output will exhibit sustained oscillations. In this method, if the system output does not exhibit the sustained oscillations, then this method does not apply. 
4.1.2. Designing PID Parameters. The transfer function of the PID controller is

$$
G_{c}(s)=K_{p}\left(1+\frac{1}{s T_{i}}+T_{d} s\right) .
$$

The objective is to achieve a unit step response curve of the designed system that exhibits a maximum overshoot of $25 \%$. If the maximum overshoot is excessive, for example, greater than $40 \%$, fine tuning should be done to reduce it to less than $25 \%$.

From Ziegler-Nichols frequency method of the second method, the table suggested tuning rule according to the formula shown. From these we are able to estimate the parameters of $K_{p}, T_{i}$, and $T_{d}$.

The values of the PID parameters $K_{p}, T_{i}$, and $T_{d}$ will be

$$
\begin{aligned}
& K_{p}=0.6 K_{e r}=9.612, \\
& T_{i}=0.5 \times 0.557=0.2785, \\
& T_{d}=0.125 \times 0.557=0.0 .06925 .
\end{aligned}
$$

4.2. Genetic Algorithm. God is the creator of the whole universe. Ever since its creation evolution has been a part and parcel of its functioning. New organisms have evolved from their ancestors; and this evolution is governed by a simple law which Charles Darwin named as "Survival of the Fittest."

Genetic algorithms are search algorithms based on natural selection and natural genetics. They combine survival of fittest among structures with structured yet randomized information exchange to form a search algorithm. Genetic algorithm has been developed by John Holland and his coworkers in the University of Michigan in the early 1960s. Genetic algorithms are theoretically and empirically proved to provide robust search in complex spaces. Its validity in Function Optimization and Control Applications is well established. Genetic algorithms (GA) provide a general approach for searching for global minima or maxima within a bounded, quantized search space. Since GA only requires a way to evaluate the performance of its solution guesses without any prior information, it can be applied generally to nearly any optimization problem. GA is usually extensively modified to suit a particular application. As a result, it is hard to classify a "generic" or "traditional" GA, since there are so many variants. An improvement to the "traditional" GA is to provide faster and more efficient searches for GAS that does not rely on average chromosome convergence (i.e., applications which are only interested in the best solution). The "traditional" GA is composed of a fitness function, a selection technique, and crossover and mutation operators which are governed by fixed probabilities. These operations form a genetic loop as shown in Figure 4. Since the probabilities are constant, the average number of local and global searches in each generation is fixed. In this sense, the GA exhibits a fixed convergence rate and therefore will be referred to as the fixedrate $\mathrm{GA}$.

A simple GA (Figure 3) consists of five steps.

(i) Start with a randomly generated population of $N$ chromosomes, where $N$ is the size of population and $l$ the length of chromosome $x$. (ii) Calculate the fitness value of function $\varphi(x)$ of each chromosome $x$ in the population.

(iii) Repeat until $N$ offsprings are created.

(iv) Replace current population with newly created one.

(v) Go to Step (ii).

4.2.1. Designing of PID Using Genetic Algorithm. GA can be applied to the tuning of PID controller gains to ensure optimal control performance at nominal operating conditions.

It is good to discuss the differences between genetic algorithm and the traditional methods. This will help us understand why GA is more efficient than the latter. Genetic algorithms are substantially different to the more traditional search and optimization techniques. The five main differences are as follows.

(1) Genetic algorithms search a population of points in parallel, not from asingle point.

(2) Genetic algorithms do not require derivative information or auxiliary knowledge; only the objective function and corresponding fitness levels influence the direction of the search.

(3) Genetic algorithms use probabilistic transition rules, not deterministic rules.

(4) Genetic algorithms work on an encoding of a parameter set not the parameter set itself (except where realvalued individuals are used).

(5) Genetic algorithms may provide a number of potential solutions to a given problem and the choice of the final is left up to the user.

4.2.2. Results of the Implemented Genetic Algorithm PID Controller. In the following section, the results of the implemented genetic algorithm PID controller will be analyzed. The GA designed PID controller is initially initialized with population size of 5 and the response analyzed. It was then initialized with population sizes of $10,15,20$, and 25 . The response of the GA designed PID will then be analyzed for the smallest overshoot, fastest rise time, and the fastest settling time. The best response will then be selected as shown in Figure 5.

From the following responses, that is, by comparing the graphs shown in Figures 2 and 5, we can observe that GA designed PID will have better response compared to the Z$\mathrm{N}$ tuned PID Method.

The GA designed PID is much better since it has no overshoot but it suffers in terms of rise time and settling time.

4.3. LQG Optimal Controller. The LQG control signal $u$ is a state feedback described below:

$$
u=-K x,
$$




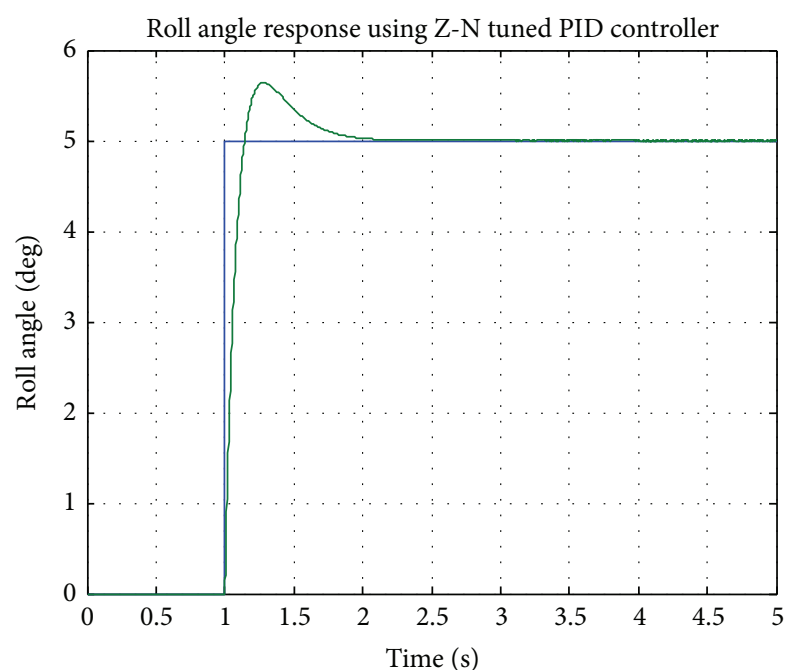

FIGURE 2: $\phi$ versus $a_{y}$ error transient response for Z-N PID controller.

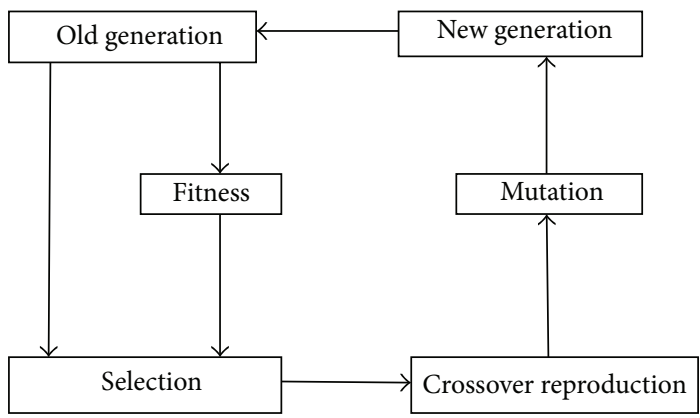

FIGURE 3: Block diagram of GA.

where the vector $K$ is obtained from the solution of a Riccati algebraic equation. $u$ can be derived from the minimization of the quadratic cost function:

$$
J=\sum_{k=0}^{\infty}\left[x^{T}(k) q_{c}(k) x(k)+u^{T}(k) r_{c}(k) u(k)\right],
$$

where $q_{c}(k)$ and $r_{c}(k)$ are weight matrices, that is, design parameters chosen to meet the desired closed-loop performance. Equation (15) is a general quadratic cost function which not only states excursions but controls excursions and state-control products as well. This equation plays a significant role in designing linear optimal controller.

Thus, appropriate selection of the weighting matrix $Q$ through $Q_{11}$ is crucial in the design of optimal regulators with prescribed closed-loop poles. The selection of weighting matrix $R>0$ is arbitrary from this point of view, and $R$ could be used as a scaling factor for the input channels. Scaling $R$ for single-input systems has no effect, since it will only result in the same amount of scaling on $Q$. Also $Q$ should be positivesemidefinite and $R$ positive-definite, respectively.

Thus

$$
Q=\left[\begin{array}{cc}
10 & 0 \\
0 & 10
\end{array}\right], \quad R=0.1
$$

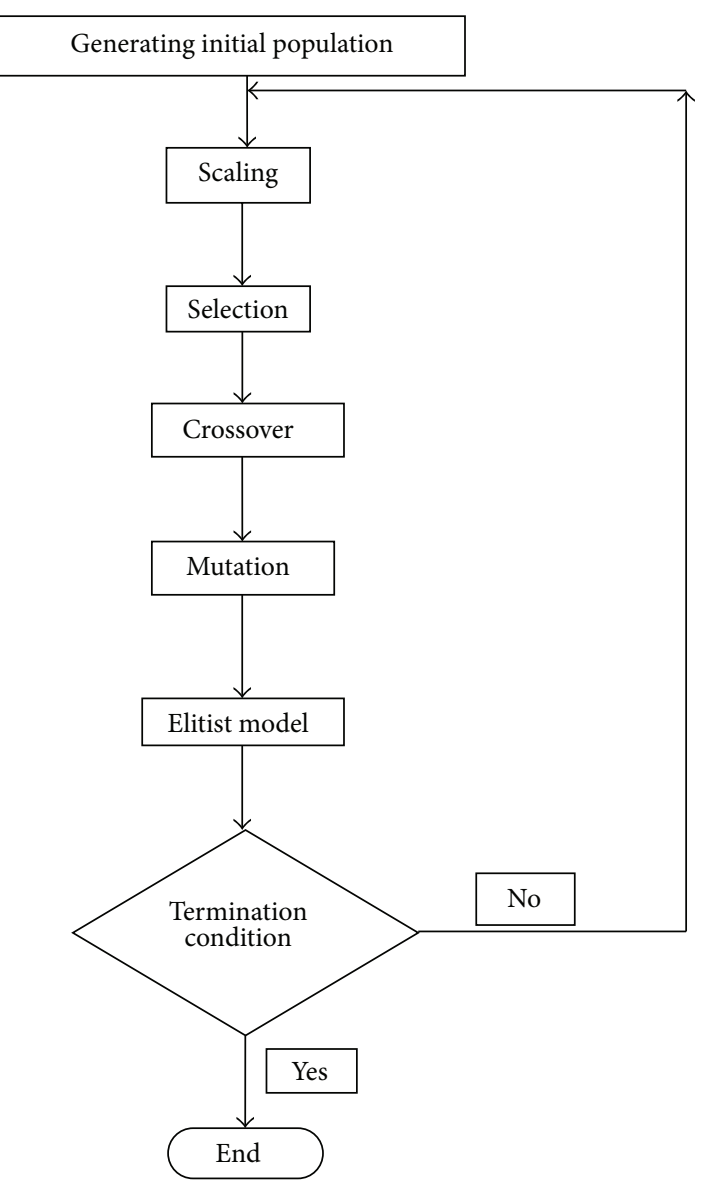

FIgURE 4: Flow chart of genetic algorithm.

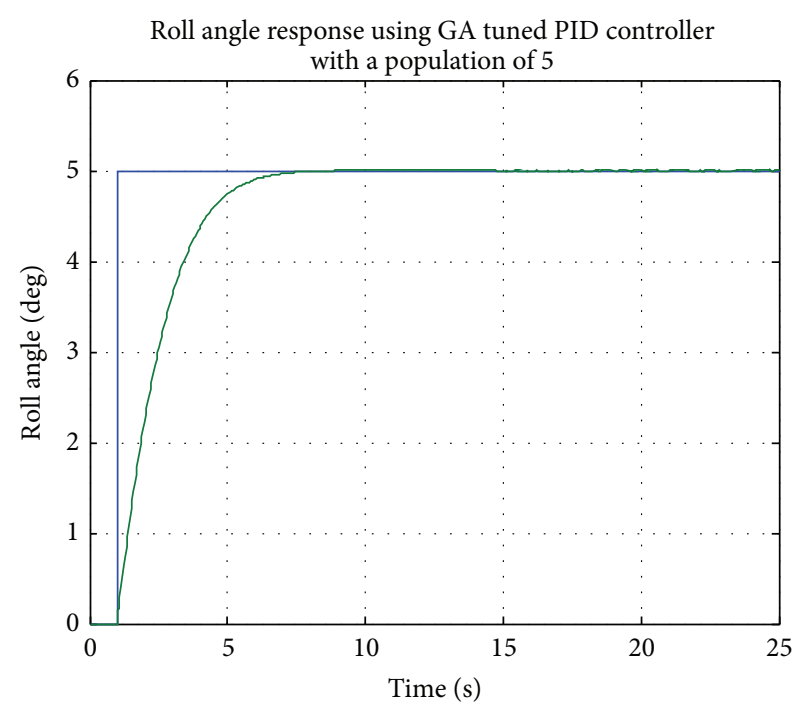

FIGURE 5: $\phi$ versus $a_{y}$ error transient response for GA tuned PID controller.

4.4. Design of a Linear Quadratic Gaussian (LQG) Controller. Design of a linear quadratic Gaussian (LQG) controller for the roll model mostly focuses on three parts based on the 


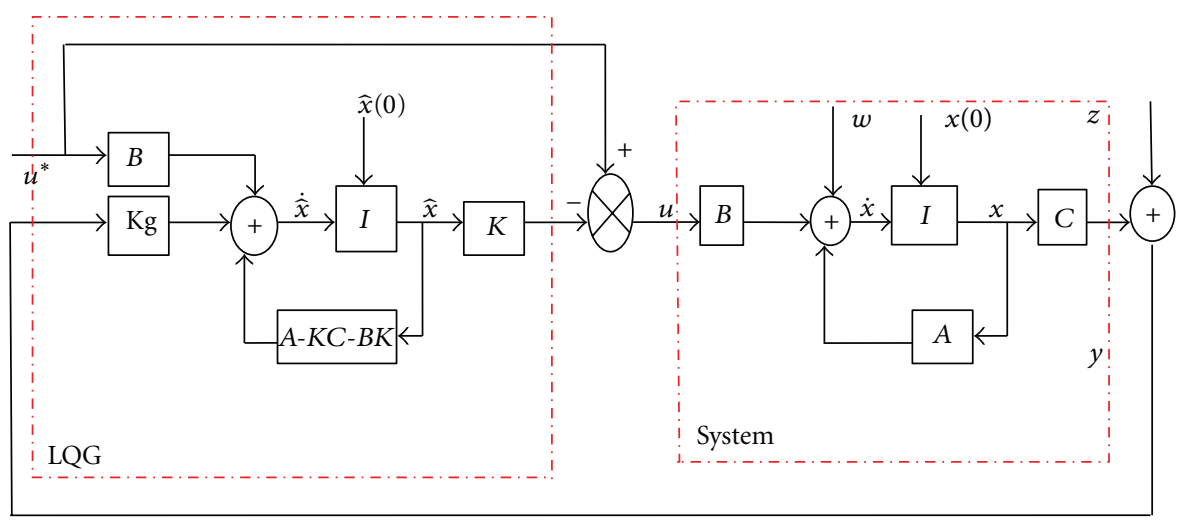

$(\mathrm{Kg}):$ Kalman gain
$(K):$ state feedback matrix
$(Y)$ : measured control output
$(u)$ : control input
$\left(u^{*}\right)$ : external input
$(I)$ : integrator

FIgURE 6: Configuration of the LQG system. basic principle of the LQG controller mentioned above: (1) design of an optimal linear quadratic estimator (LQE); (2) design of an optimal linear quadratic Gaussian regulator (LQR); (3) system integration. Together with the linear quadratic estimator the linear quadratic Gaussian regulator (LQR) solves this linear quadratic Gaussian control problem. Figure 6 shows the configuration of the LQG system.

The calculation of the LQG regulator requires the calculations (i) of the optimal state feedback gain $K$, which is received as a result of the minimization procedure (16), and (ii) of the Kalman estimator.

The LQG regulator was computed on the basis of the balancing theory and Kalman estimator.

(i) Design of a Linear Quadratic Estimator. The Kalman filter is a feasible estimation approach that can fuse multiple sensory measurements to provide relatively accurate results. The Kalman filter, in a way, can minimize the mean of the squared error from a series of noisy measurements. The Kalman filter provides an optimal recursive data processing algorithm which works in the way that the present estimated value of the state is determined by the previous estimated value of the state and the present measured data instead of the entire measured data. The Kalman filter addresses the general problem of trying to estimate the state of a discrete-time controlled process that is governed by the linear stochastic difference equation.

\section{(ii) Design of Linear Quadratic Regulator}

(a) Optimization Method. The control strategy is characterized by the control configuration illustrated in Figure 6 . The realization of the optimal control strategy depends on the state estimation and the control law. Considering the roll model control problem, the control law is optimized by minimizing the cost function to guarantee control objectives

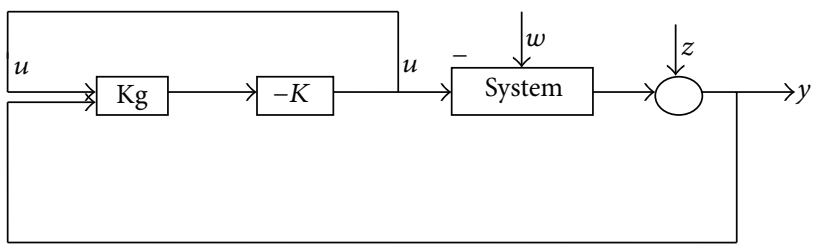

$(u)$ : control input $\quad(w)$ : process noise

$(\mathrm{Kg})$ : Kalman gain $\quad(z)$ : measurement noise

$(K)$ : LQG optimal gain $\quad(y)$ : measurement

FIgURE 7: System closed-loop configuration.

which are a minimal position error and using as little control effort as possible.

(b) Control Law. The control values can be obtained by the optimization method mentioned above. In order to calculate this, the steepest descent method was applied, which is an iterative method, being of simple implementation, but with slow convergence.

(c) Calculation and Simulation Results. The Matlab codes were made and simulation has been conducted to (1) select the controller's parameters, like the cost function's sensitivity to dynamic effects and the stop criterion, and (2) obtain the cost function and the control law. The cost function has been close to zero after 5 iterations. Furthermore, a larger contribution for the solution takes always place in the first iterations a small contribution at the end. It is noticed that the control law decreases linearly with time.

(d) System Integration. System integration is a significant step in designing LQG controller after designing the optimal estimate and configurating the optimal controller. Figure 7 shows the system closed-loop configuration. 


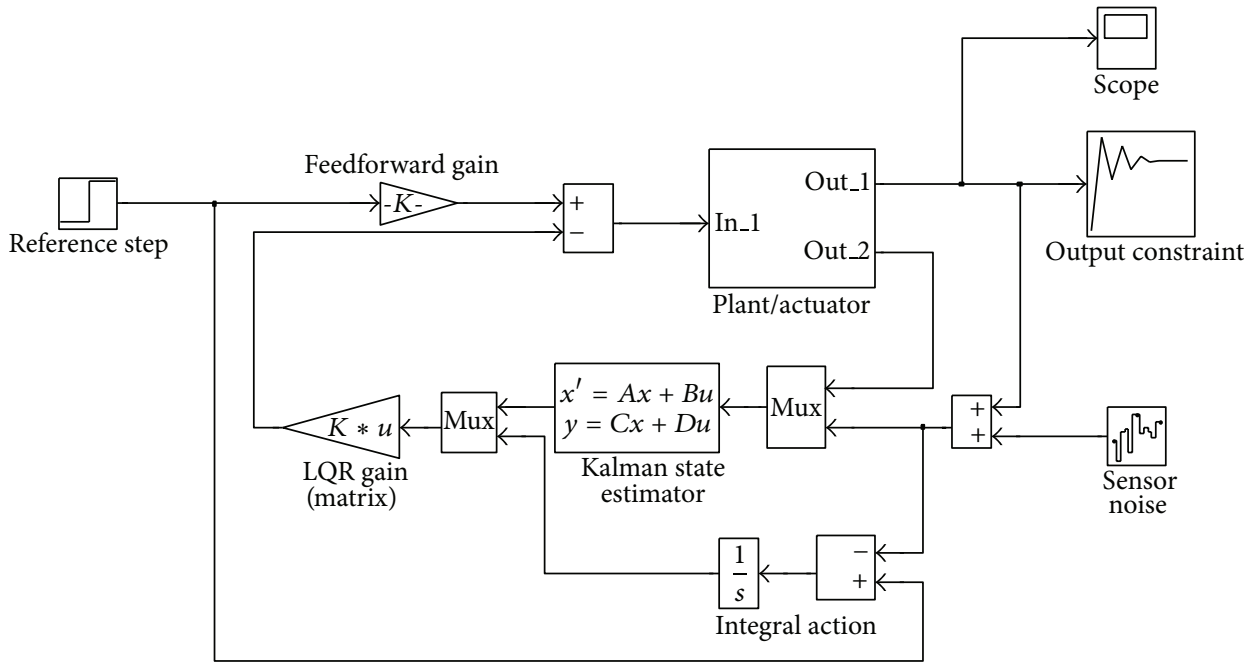

FIGURE 8: Simulation diagram for roll model.

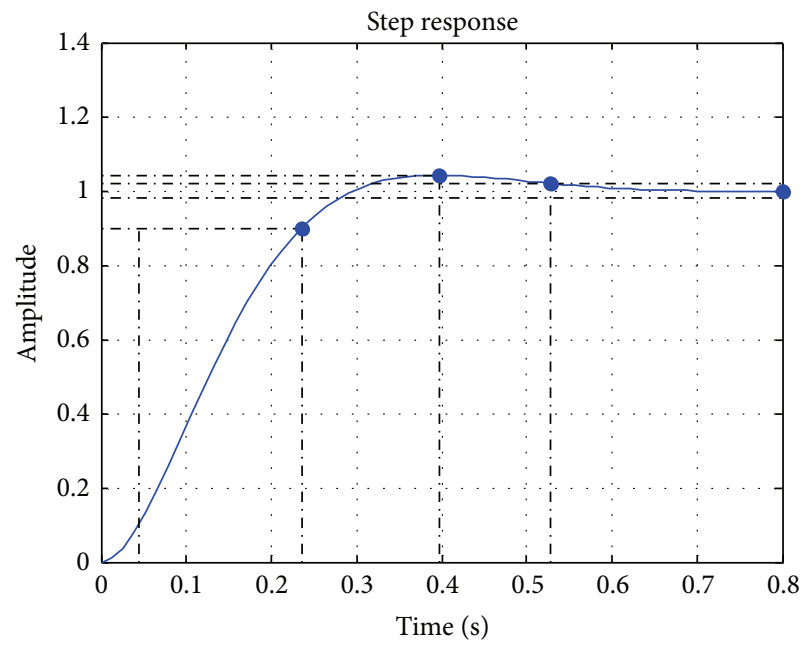

FIGURE 9: $\phi$ versus $a_{y}$ transient response for $\xi=0.71$.

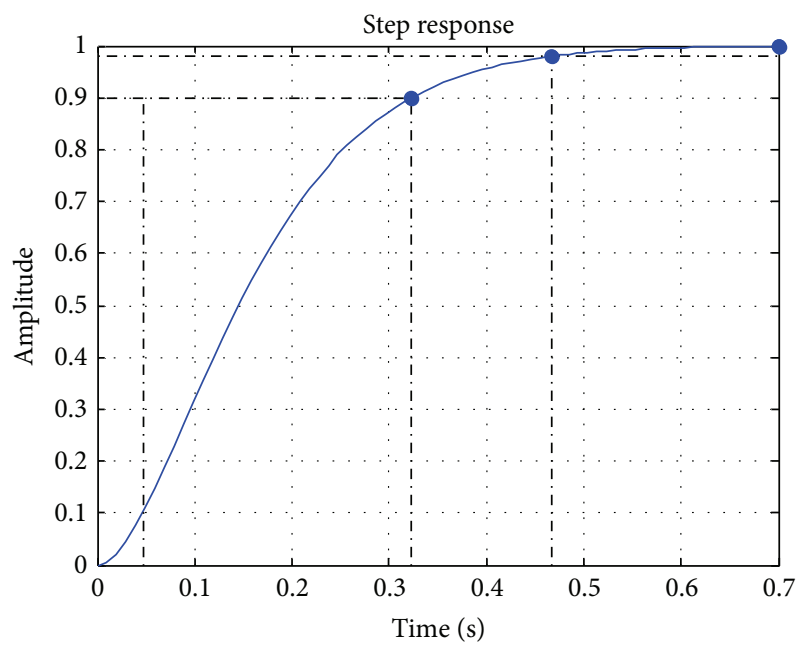

FIGURE 10: $\phi$ versus $a_{y}$ transient response for $\xi=0.95$.

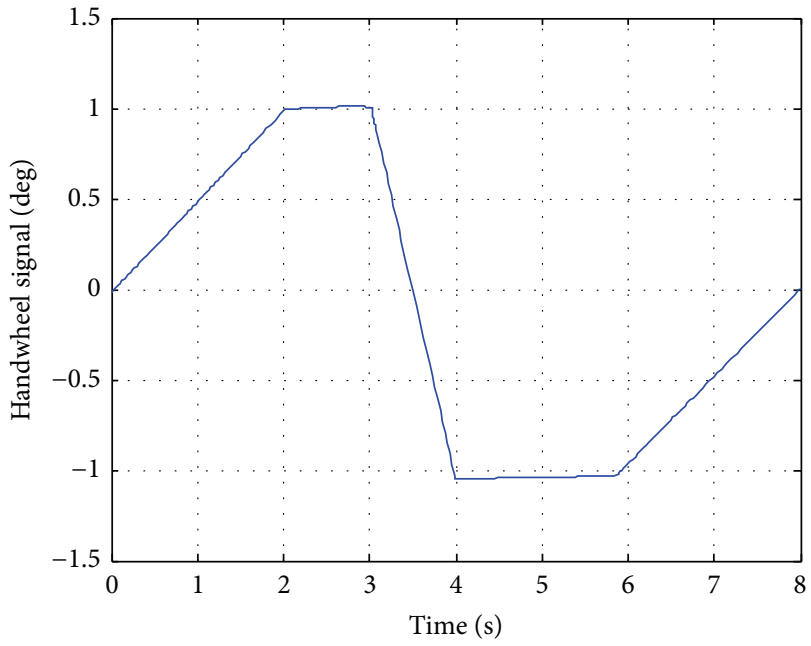

FIGURE 11: Fishhook la maneuver.

\section{Simulation Results}

In order to verify the proposed mitigation control, simulation tests are conducted according to NHTSA's Fishhook la test description, and the steering maneuver patterns are shown in Figure 11 and the simulink diagram for roll model is shown in Figure 8.

Note that the wild fluctuations occur when the handwheel angle rate changes rapidly, especially within the time ranges of [ $2 \mathrm{~s}, 3 \mathrm{~s}]$ and $[4 \mathrm{~s}, 6 \mathrm{~s}]$.

In theory, the overshoot is nearby zero if the damping ratio equals one. However, as we know, high roll stiffness and roll damping cost vehicle ride comfort. For a balance purpose, we assigned the compromised damping ratio $\xi$ at a normal factory-set value.

There are many factors that can influence the roll stiffness $k_{\Phi}$ and the roll damping $c_{\Phi}$, such as springs, dampers, and bushings. 


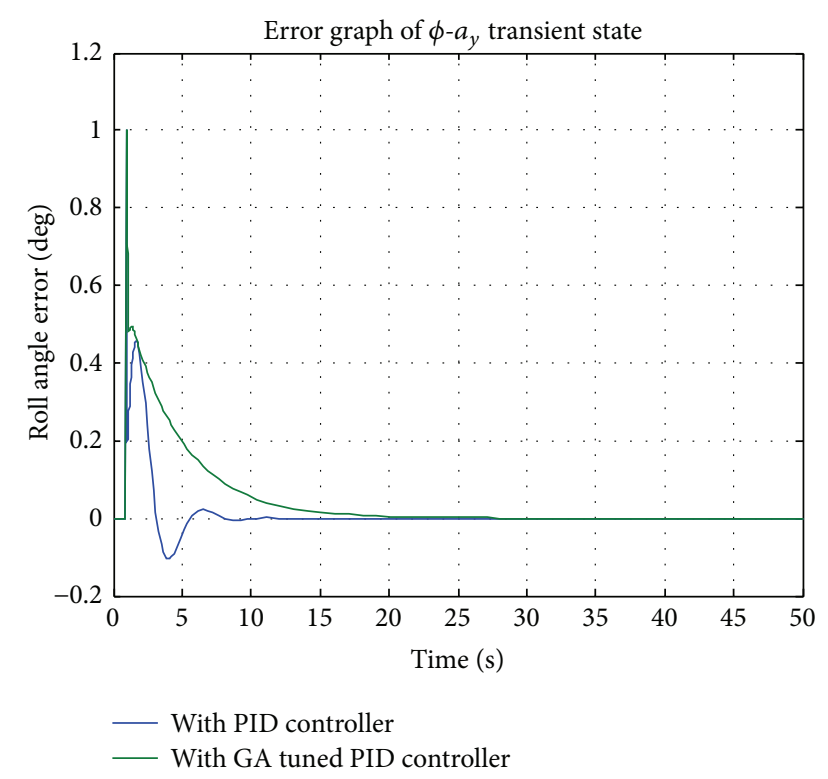

FIGURE 12: $\phi$ versus $a_{y}$ error transient response for different controllers.

TABLE 2: Results of Z-N tuned PID controller, GA tuned PID controller, and LQG controller.

\begin{tabular}{lccc}
\hline $\begin{array}{l}\text { Measuring } \\
\text { factors }\end{array}$ & $\begin{array}{c}\text { Z-N tuned PID } \\
\text { controller }\end{array}$ & $\begin{array}{c}\text { GA tuned PID } \\
\text { controller }\end{array}$ & $\begin{array}{c}\text { LQG } \\
\text { controller }\end{array}$ \\
\hline $\begin{array}{l}\text { Rise time in sec } \\
\text { Maximum }\end{array}$ & 1.1 & 5 & 0.3 \\
$\begin{array}{l}\text { overshoot in \% } \\
\text { Settling time in } \\
\text { sec }\end{array}$ & 12.5 & NA & 0.005 \\
\hline
\end{tabular}

From Table 1, and observing Figures 12 and 13, we can see that the LQG designed controller has a significant improvement over the GA designed controller.

\section{Conclusion}

The intent of this paper is to present a methodology for predicting when a vehicle may be at risk for wheel-lift prior to sliding. Additionally, multiple control strategies were presented that use the information derived from this method to mitigate vehicle rollover. The wheel-lift prediction methodologies give a sense of a vehicle's dynamic rollover propensity, provide insight into the roll dynamics of the vehicle that may not be garnered from examination of the response from the individual states alone, and may lead to better automotive designs in regard to roll dynamics.

To investigate the vehicle transient and steady states, an improved rollover model was established in this study. Based on the model, we further develop a method to limit the overshoot by adjusting the relationship between the spring stiffness and the damper damping coefficient. Additional findings include the mitigation control on vehicle stabilization, and the control logic was verified by a series of simulation tests using a midsize SUV model. Simulation

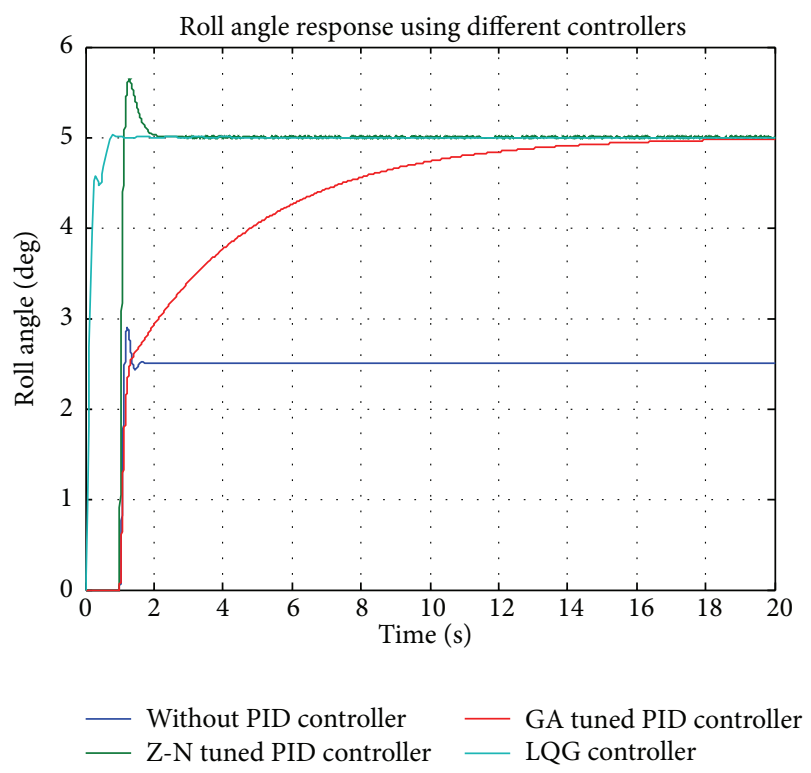

FIGURE 13: $\phi$ versus $a_{y}$ error transient response for different controllers.

results indicated a decreased overshoot of the roll angle and a better confined steady value. The adjusting method proposed in this paper may help with the design of both passive and active suspension controls to increase vehicle stability. We are confident that, with additional studies, the proposed model will be applicable for a real vehicle in the near future.

An optimal controller for a roll model has been designed in Matlab simulation to implement a predictive control to the vehicle rollover. The Kalman estimate was applied to determine the system output and the numeric optimization method was used to optimize the cost function, which enables a tradeoff between the regulation performance and the control effort. Process disturbance and measurement noises were taken into account in controller design.

The GA designed PID is much better than Z-N based PID controller since it has no overshoot but it suffers in terms of rise time and settling time.

From Table 2, we can see that the LQG designed controller has a significant improvement over the GA designed controller.

\section{Nomenclature}

$v_{x}$ : Longitudinal velocity (body-fixed frame)

$\omega_{r}$ : Yaw rate (angular rate about vertical axis)

$m$ : Total mass of the vehicle

$I_{z z}$ : Inertia about the vertical axis

a: Front-axle-to-CG distance

$b$ : Rear-axle-to-CG distance

L: Track of vehicle

$t$ : Width of vehicle

$\beta$ : Side slip angle of the vehicle body

$k_{1}$ : Front cornering stiffness

$k_{2}$ : Rear cornering stiffness

$\phi$ : Roll angle 


$\begin{array}{ll}\dot{\phi}: & \text { Roll rate } \\ \ddot{\phi}: & \text { Body roll acceleration } \\ a_{y}: & \text { Lateral acceleration } \\ \dot{\omega}_{r}: & \text { Yaw acceleration } \\ I_{Z}: & \text { Inertia about the yaw moment } \\ I_{X}: & \text { Roll moment of vehicle inertia } \\ \alpha_{1}: & \text { Slip angles of front tires } \\ \alpha_{2}: & \text { Slip angles of rear tires } \\ h_{0}: & \text { Height of the vehicle's center of gravity }(\mathrm{CG}) \\ & \text { standing above the ground level } \\ h: & \text { Distance between the vehicle CG and the } \\ T: & \text { assumed roll axis } \\ \Delta h \text { and } \Delta T: \begin{array}{l}\text { Deformation of suspension and tire } \\ k_{\Phi}:\end{array} & \text { Total roll stiffness } \\ c_{\Phi}: & \text { Total roll damping of suspension. }\end{array}$

\section{Conflict of Interests}

The authors declare that there is no conflict of interests regarding the publication of this paper.

\section{References}

[1] B.-C. Chen and H. Peng, "Differential-braking-based rollover prevention for sport utility vehicles with human-in-the-loop evaluations," Vehicle System Dynamics, vol. 36, no. 4-5, pp. 359389, 2001.

[2] D. J. M. Sampson and D. Cebon, "Active roll control of single unit heavy road vehicles," Vehicle System Dynamics, vol. 40, no. 4, pp. 229-270, 2003.

[3] H.-J. Kim, H. S. Yang, and Y.-P. Park, "Robust roll control of a vehicle: experimental study using a hardware-in-the-loop setup," Proceedings of the Institution of Mechanical Engineers D, vol. 216, no. 1, pp. 1-9, 2002.

[4] R. W. Goldman, M. El-Gindy, and B. T. Kulakowski, "Rollover dynamics of road vehicles: literature survey," Heavy Vehicle Systems, vol. 8, no. 2, pp. 103-141, 2001.

[5] P. Ponticel, "Dynamic testing rollover on the way," Automotive Engineering International, pp. 26-28, 2003.

[6] D. W. Shuttlewood, D. A. Crolla, and R. S. Sharp, "Active roll control for passenger cars," Vehicle System Dynamics, vol. 22, no. 5-6, pp. 383-396, 1993.

[7] J. Darling and T. J. Ross-Martin, "A theoretical investigation of a prototype active roll control system," Proceedings of the Institution of Mechanical Engineers D, vol. 211, no. 1, pp. 3-12, 1997.

[8] V. Cherian, R. Shenoy, A. Stothert, J. Shriver, J. Ghidella, and T. D. Gillespie, "Model-based Besign of a SUV anti-rollover control system," SAE Paper no. 2008-01-0579, 2008.

[9] B. Lee, A. Khajepour, and K. Behdinan, "Vehicle stability through integrated active steering and differential braking," SAE Paper 2006-01-1022, 2006.

[10] B.-C. Chen and H. Peng, "A real-time rollover threat index for sports utility vehicles," in Proceedings of the American Control Conference, pp. 1233-1237, June 1999.

[11] C. Foo, H. Yeh, S. Chang, and K. J. Schemansky, "Apparatus and method for detecting vehicle rollover having a discriminating safing function," U.S. Patent 6600414, 2001.
[12] K. Hamsch and M. Roelleke, "Method for classifying a rollover event of a vehicle," U.S. Patent 6732 034, 2004.

[13] B. Johansson and M. Gafvert, "Untripped SUV rollover detection and prevention," in Proceedings of the IEEE Conference on Decision and Control, pp. 5461-5466, Atlantis, Bahamas, December 2004.

[14] A. Hac, "Rollover stability index including effects of suspension design," SAE Paper No. 2002-01-0965, 2002.

[15] T. Shim and D. Toomey, "Investigation of active steering/wheel torque control at the rollover limit maneuver," SAE Paper No. 2004-01-2097, 2004.

[16] A. Hac, "Influence of active chassis systems on vehicle propensity to maneuver-induced rollovers," SAE Paper 2002-01-0976, 2002.

[17] S. Takano, M. Nagai, T. Taniguchi, and T. Hatano, "Study on a vehicle dynamics model for improving roll stability," JSAE Review, vol. 24, no. 2, pp. 149-156, 2003.

[18] A. Hac, T. Brown, and J. Martens, "Detection of vehicle rollover," SAE Paper 2004-01-1757, 2004.

[19] M. Tanelli, S. M. Savaresi, and C. Cantoni, "Longitudinal vehicle speed estimation for traction and braking control systems," in Proceedings of the IEEE International Conference on Control Applications (CCA '06), pp. 2790-2795, Munich, Germany, October 2006.

[20] C. S. Namuduri, M. A. Golden, and J. Praeckel, "Concurrent research and development of a magnetic ride control system," in Proceedings of the 29th Annual Conference of the IEEE Industrial Electronics Society (IECON '03), pp. 2853-2858, November 2003. 


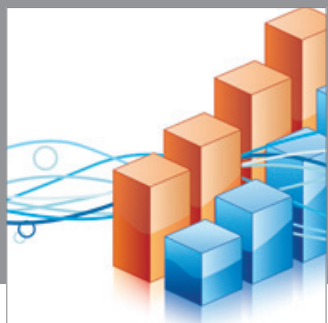

Advances in

Operations Research

mansans

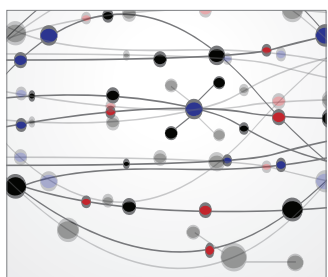

The Scientific World Journal
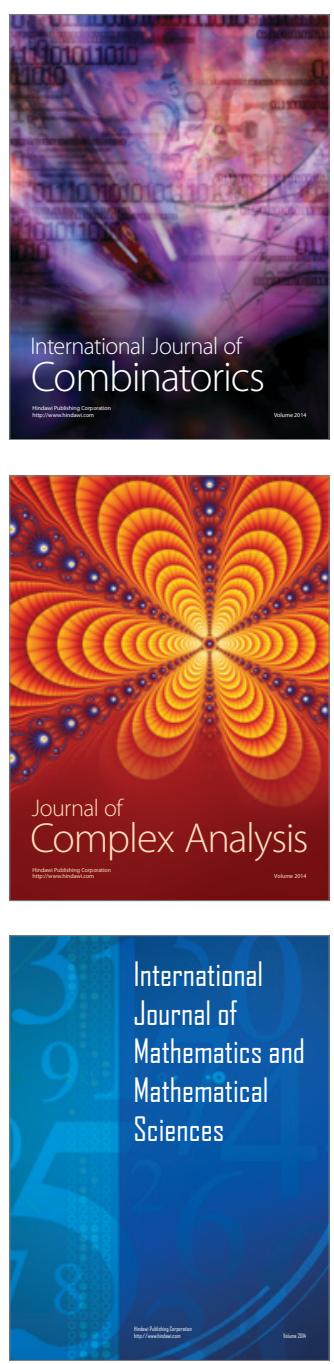
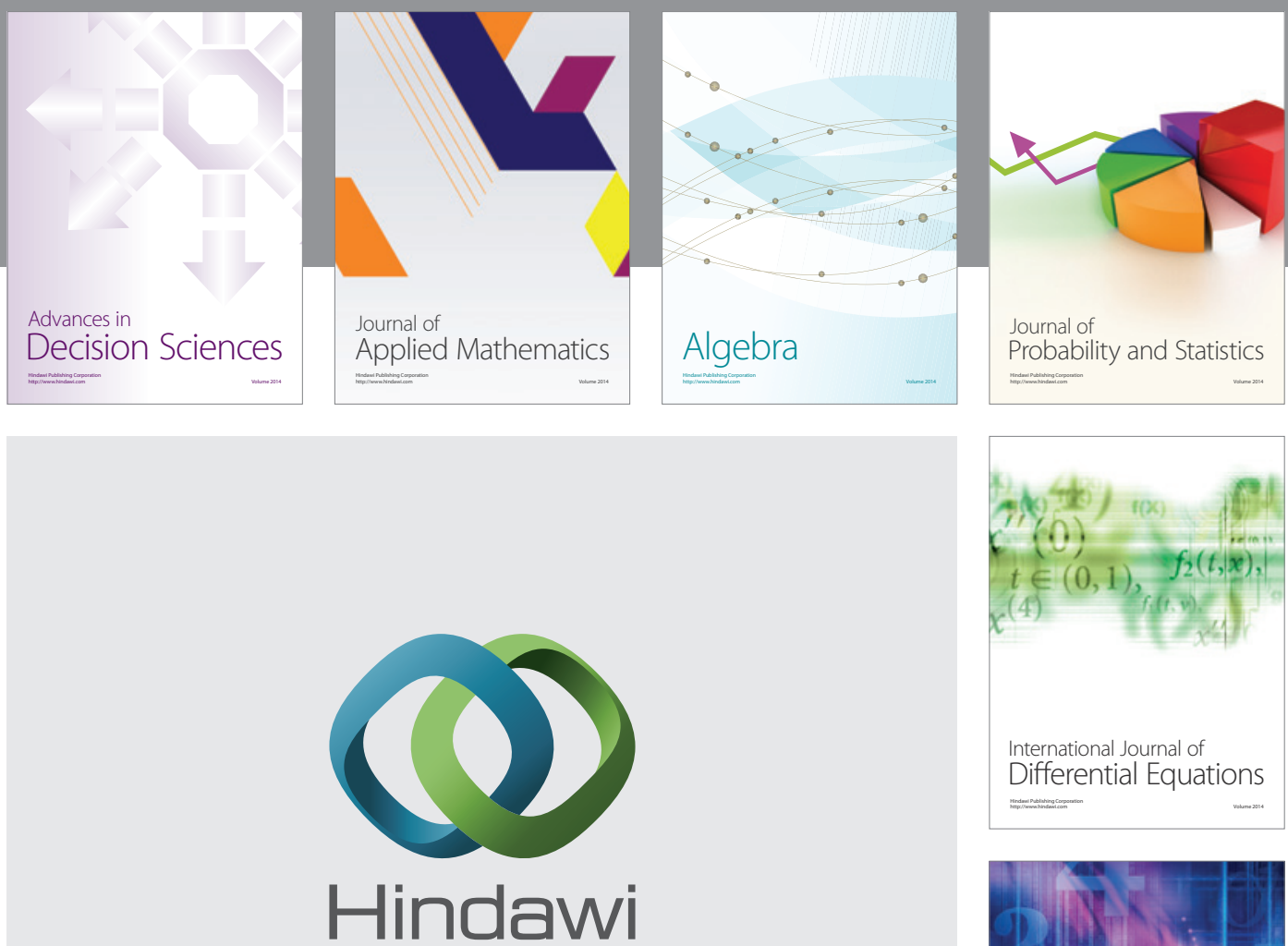

Submit your manuscripts at http://www.hindawi.com
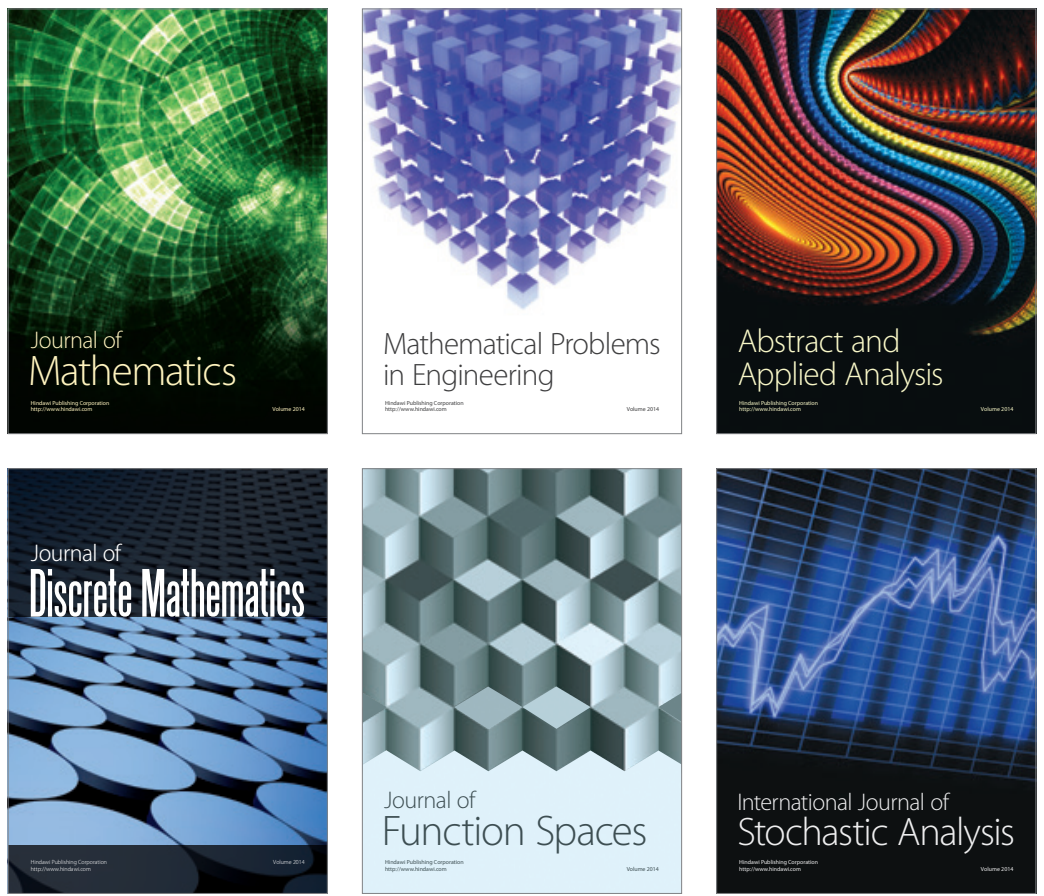

Journal of

Function Spaces

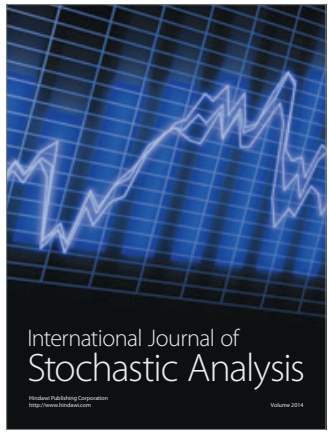

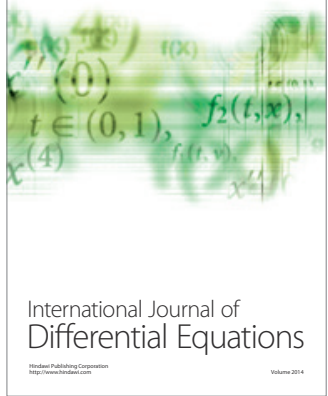
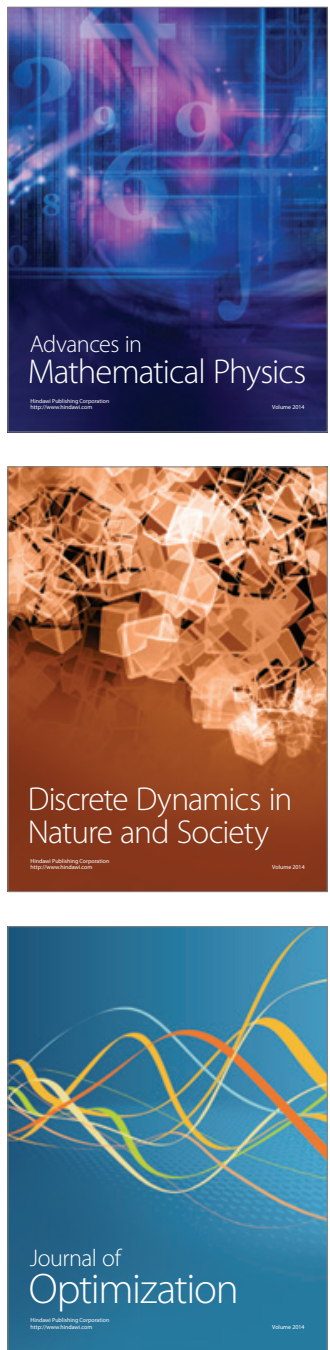